\title{
Blockspin Scheme and Cluster Algorithm for Quantum Spin Systems
}

\author{
U.-J. Wiese ${ }^{\mathrm{a}}$ and H.-P. Ying ${ }^{\mathrm{b} *}$ \\ anstitut für Theoretische Physik, Universität Bern, Sidlerstrasse 5, CH-3012 Bern, Switzerland \\ bZhejiang Institute of Modern Physics, Zhejiang University, Hangzhou 310027, P.R.China
}

We present a numerical study using a cluster algorithm for the 1 -d $S=1 / 2$ quantum Heisenberg models. The dynamical critical exponent for anti-ferromagnetic chains is $z=0.0(1)$ such that critical slowing down is eliminated.

\section{INTRODUCTION}

Since 1976 it is well-known that several mappings of the partition function of a $d$-dimensional $S=1 / 2$ quantum spin model onto a $(d+1)$ dimensional classical spin model exist [1,2]. The maps lead to a Feyman path integral formula of quantum statistical mechanics. The equivalence opens the possibility to use powerful computational techniques to obtain information on quantum spin systems. Monte Carlo (MC) simulation, a method to get precise numerical data, has played an important role in the studies [3], and has especially been applied to the antiferromagnetic models [4.5] since Haldane's conjecture [6] and the discovery of high- $T_{c}$ superconductors [7]. There are, however, some problems with the standard numerical methods, which use importance-sampling techniques for the $(d+1)$ dimensional induced classical spin systems which have multi-spin interactions. Their effect is characterized by large autocorrelation times (in units of MC sweeps needed to create a new statistically independent configuration) in the low temperature region and in the euclidean time continuum limit. In this paper a cluster algorithm is developed for simulations of quantum spin systems [8], especially of 1-d quantum Heisenberg models, after mapping them to 2-d induced classical spin models and using a blockspin scheme.

\footnotetext{
*speaker at the conference
}

Figure 1. Heisenberg magnet on a chain with an even number of spins $2 N$.

\section{METHOD}

Consider for definiteness a 1-d $S=1 / 2$ Heisenberg model defined on a chain with an even number of spins $L=2 N$ (fig.1) with periodic boundary conditions. The Hamilton operator is given by

$H=\frac{J}{4} \sum_{i=1}^{L} \vec{\sigma}_{i} \cdot \vec{\sigma}_{i+1}$

Here $\vec{\sigma}_{i}$ is a Pauli spin operator at the point $i$ on the chain. $J>0$ corresponds to an antiferromagnet (AF), while $J<0$ corresponds to a ferromagnet $(\mathrm{F})$. Using the checkerboard decomposition $H=H_{1}+H_{2}$ (fig.1) and the Trotter formula [3], the partion function is expressed as a path integral given by

$$
Z=\operatorname{Tr} \exp (-\beta H)=\prod_{x, t} \sum_{S(x, t)= \pm 1} \exp (-S),
$$

where $\beta$ is the inverse temperature. Eq.(2) is equivalent to a 2 -d classical spin model on a rect- 
angular lattice $L \times 2 M$ with Ising-like variables $s(x, t)= \pm 1$. The lattice spacing in the additional so-called euclidean time or Trotter direction is $\epsilon=1 / M$ (fig.2). The classical spins interact with each other via 4 -spin couplings $\exp \left(-S\left[s_{1}, s_{2}, s_{3}, s_{4}\right]\right)=\left\langle s_{1}, s_{2}\right| \exp \left(-\epsilon \beta J \vec{\sigma}_{i}\right.$. $\left.\vec{\sigma}_{i+1} / 4\right)\left|s_{3}, s_{4}\right\rangle$, which are given by elements of the transfer matrix. For the AF one has

$$
\begin{gathered}
S[1,1,1,1]=S[-1,-1,-1,-1]=\epsilon \beta J / 4, \\
S[1,-1,1,-1]=S[-1,1,-1,1]= \\
\epsilon \beta J / 4-\log [(\exp (\epsilon \beta J)+1) / 2], \\
S[1,-1,-1,1]=S[-1,1,1,-1]= \\
\epsilon \beta J / 4-\log [(\exp (\epsilon \beta J)-1) / 2] .
\end{gathered}
$$

All other action values are infinite and the corresponding elements of the transfer matrix are zero. The matrix elements can be interpreted as the Boltzmann weights of the spin configurations. Up to here the quantum spins $\vec{\sigma}_{i}$ with 2 -spin couplings living on a 1-d chain have been mapped to classical spins $s(x, t)$ with 4 -spin couplings living in the 2-d plane. However, most classical spin configurations are forbidden, because for them some elements of the transfer matrix are zero (their Boltzmann factor vanishes). Therefore, it is natural to attempt to choose a collective nonlocal update technique. For models with 2-spin couplings this can be done using the SwendsenWang [9] or Wolff [10] cluster algorithms which flip whole cluster of spins simultaneously. These algorithms, however, can not be applied directly to a model with 4-spin couplings.

To make an application of the cluster technique possible we further map the classical spin model with 4 -spin couplings $S\left[s_{1}, s_{2}, s_{3}, s_{4}\right]$ to a blockspin model. A blockspin $B(n, m)=$ $\{s(x, t), s(x+1, t), s(x, t+1), s(x+1, t+1)\}$ is a collection of four spins, where $x=2 n-1, t=$ $2 m$. The blockspins interact via 2-blockspin interactions $S\left[B, B^{\prime}\right]=S\left[s_{1}, s_{2}, s_{3}, s_{4}\right]$ as shown in fig.2a. Each spin belongs exactly to one blockspin and the blockspins live on a lattice with a doubled lattice spacing. The spins can also be arranged to blockspins in another way, $\bar{B}(n, m)=$ $\{s(x, t), s(x+1, t), s(x, t+1), s(x+1, t+1)\}$ with $x=2 n, t=2 m-1$ (see fig. $2 \mathrm{~b}$ ). An updating algorithm for the blockspins must alternate between
Figure 2. 2-d induced classical spin system with 4 -spin couplings depicted by shaded plaquettes. (a) The scheme $\{B\}$ : each blockspin consists of 4 spins and interacts with its nearest-neighbors via the original 4 -spin interaction. (b) the other scheme $\{\bar{B}\}$ which is dual to the scheme $\{B\}$.

the schemes $\{B\}$ and $\{\bar{B}\}$ to ensure ergodicity. We have also chosen two kinds of nonlocal blockspins [8] to change the magnetization and the socalled winding number by odd integers. The cluster algorithm makes use of a flip symmetry of the blockspin model. A blockspin $B=\left\{s_{1}, s_{2}, s_{3}, s_{4}\right\}$ is flipped to $-B=\left\{-s_{1},-s_{2},-s_{3},-s_{4}\right\}$ simply by flipping all spins in $B$. The blockspin action $S\left[B, B^{\prime}\right]$ is invariant under a flip of all blockspins because the original action is symmetric against flipping all spins.

\section{MONTE CARLO RESULTS}

We now describe how to generate transitions between blockspin configurations. a) The algorithm puts bonds between all nearest-neighbor pairs of blockspins with a probability $p=$ $\left.1-\min \left\{1, \exp \left(-S\left[-B, B^{\prime}\right]\right) / \exp \left(-S\left[B, B^{\prime}\right]\right)\right\} . b\right)$ Two blockspins belong to the same cluster if they are connected by a bond. c) All blockspins in one cluster must be flipped simultaneously. d) Us- 
algorithms we measure the autocorrelation functions $C_{\mathcal{O}}(t)$, where $\mathcal{O}$ denotes the observables, i.e. the internal energy $e$, and the uniform and staggered susceptibilities $\chi$ and $\chi_{s}$. Then we obtain the integrated autocorrelation times $\tau_{\mathcal{O}}$ by

$\exp \left(-1 / \tau_{\mathcal{O}}\right)=\sum_{t=1}^{\infty} C_{\mathcal{O}}(t) / \sum_{t=0}^{\infty} C_{\mathcal{O}}(t)$.

In all the simulations we have performed a random start followed by 5000 sweeps for thermalization and 50000 sweeps for measurements using the single-cluster updating. From the data at $\beta J=1$ shown in fig. 3 we obtain the dynamical critical exponent $z_{e}$ both for the anti-ferromagnet and for the ferromagnet. $z_{e}$ is defined in the continuum limit $\epsilon \rightarrow 0$ by $\tau_{e} \propto 1 / \epsilon^{z}$. We find

$z_{e}=0.0(1)$ for the cluster algorithm,

$z_{e}=0.8(1)$ for the Metropolis algorithm.(5)

The autocorrelation times $\tau_{\chi}$ and $\tau_{\chi_{s}}$ do not diverge in the continuum limit even for the Metropolis algorithm. Still, the corresponding autocorrelation times are at least an order of magnitude larger than the ones of the cluster algorithm.

As shown in fig.4, the Metropolis algorithm has severe problems with slowing down at low temperatures. At $\beta J=8$ for example $\tau_{\chi_{s}}=12(1)$ for $J>0$, and $\tau_{\chi}=3300(200)$ for $J<0$. The cluster algorithm, on the other hand, has autocorrelation times of at most a few sweeps and it can update the configurations efficiently at lower temperatures like e.g. $\beta J=16$. In fig. 4 the $\tau_{\chi}$ and $\tau_{\chi_{s}}$ are fitted by

$\tau_{\chi} \propto \exp \left(c_{\chi} \beta|J|\right), \quad \tau_{\chi_{s}} \propto \exp \left(c_{\chi_{s}} \beta|J|\right)$.

ing the Swendsen-Wang multi-cluster method [9] each cluster is flipped independently with probability $1 / 2$. For the Wolff single-cluster algorithm 10 one blockspin is randomly chosen and the cluster to which it belongs is flipped. The procedure $a)-d$ ) of changing a configuration satisfies the detailed balance condition [10]. For ergodicity we alternate between the $\{B\}$ and $\{\bar{B}\}$ schemes. We tested the blockspin cluster algorithm in detail for one-dimensional spin chains. To demonstrate the efficiency of the algorithm we compare it to a blockspin Metropolis update. For both

The data at $\beta J=16$ show some slowing down for the ferromagnet, but the autocorrelation times are moderate. For anti-ferromagnet there is no indication of slowing down such that

$z_{e}=z_{\chi}=z_{\chi_{s}}=0.0(1)$.

\section{DISCUSSION}

First, we like to mention a few words about frustration. As it is well known the Swendsen- 
Figure 4. (a) The plot of $\log \tau_{\chi_{s}}$ versus $\beta J$ for AF couplings and $(b) \log \tau_{\chi}$ for $\mathrm{F}$ couplings with $2 N=128$ and $\epsilon=\beta / M=0.125$.

Wang and Wolff cluster algorithms eliminate critical slowing down for the Ising and Potts models because they are not frustrated. For strongly frustrated models, e.g. for spin glasses, cluster algorithms do not work efficiently. Therefore the question arises if our blockspin models are frustrated or not. We have verified that the blockspin model for 1-d AF couplings has indeed no frustrated allowed configurations. For the 1-d ferromagnet, on the other hand, some allowed configurations have a weak frustration. However, in the continuum limit $\epsilon \rightarrow 0$ the frustration disappears.
We believe that the weak frustration is responsible for the mild slowing down in the ferromagnetic case at low temperature.

The blockspin cluster algorithm can also be applied to other models, e.g. to systems with anisotropic couplings or in an external magnetic field, to models of higher spins $(s=1,3 / 2, \ldots)$, to six- and eight-vertex models and to 1 -d fermion systems. Work on the 2-d anti-ferromagnet is in progress 11.

We are grateful to P. Hasenfratz for useful suggestions and discussions. This work was supported partly by the Schweizer Nationalfond. One of us (HPY) would like to acknowledge the support from the PAO's scholarship for CSSA.

\section{REFERENCES}

1. M. Suzuki, Prog. Theor. Phys. 56 (1976) 1454; Comm. Math. Phys. 51 (1976) 183.

2. J. J. Cullen and D. P. Landau, Phys. Rev. B27 (1983) 297.

3. H. De. Raedt and A. Lagendijk, Phys. Rep. 127 (1985) 233.

4. M. Uchinami, Phys. Rev. B39 (1989) 4554; K. Normura, Phys. Rev. B40 (1989) 2421.

5. T. Barnes, Int. J. Mod. Phys. C2(1991)659; M. S. Makivic and H.-Q. Ding, Phys.Rev. B43 (1991) 3562.

6. F. D. M. Haldane, Phys. Rev. Lett. 50 (83) 1153; Phys. Lett. 93A (1983) 464.

7. G. Bednorz and K. A. Müller, Z. Phys. B64 (1986) 189.

8. U.-J. Wiese and H.-P. Ying, Phys. Lett. 168A (1992) 143.

9. R. Swendsen and J.-S. Wang, Phys. Rev. Lett. 58 (1987) 86.

10. U.Wolff, Phys. Rev. Lett. 62 (1989) 361; Nucl. Phys. B334 (1990) 581.

11. U.-J. Wiese and H.-P. Ying, in preparation. 